\title{
Fixed Point Results for $\alpha-\psi$-Contractive Mappings Including Almost Contractions and Applications
}

\author{
Gonca Durmaz, Gülhan Minak, and Ishak Altun \\ Department of Mathematics, Faculty of Science and Arts, Kirikkale University, 71450 Yahsihan, Kirikkale, Turkey \\ Correspondence should be addressed to Gonca Durmaz; gncmatematik@hotmail.com
}

Received 29 May 2014; Accepted 9 July 2014; Published 22 July 2014

Academic Editor: Salvador Romaguera

Copyright (C) 2014 Gonca Durmaz et al. This is an open access article distributed under the Creative Commons Attribution License, which permits unrestricted use, distribution, and reproduction in any medium, provided the original work is properly cited.

\begin{abstract}
In the recent paper (B. Samet, C. Vetro, and P. Vetro, Fixed point theorems for $\alpha$ - $\psi$-contractive type mappings, Nonlinear Analysis. Theory, Methods and Applications, 75 (2012), 2154-2165.), the authors introduced the concept of $\alpha$-admissible maps on metric spaces. Using this new concept, they presented some nice fixed point results. Also, they gave an existence theorem for integral equation to show the usability of their result. Then, many authors focused on this new concept and obtained a lot of fixed point results, which are used for existence theorems. In this paper, we not only extend some of the recent results about this direction but also generalize them. Then, we give some examples to show our results are proper extensions. Furthermore, we use our results to obtain the existence and uniqueness result for a solution of fourth order two-point boundary value problem.
\end{abstract}

\section{Introduction and Preliminaries}

Fixed point theory contains many different fields of mathematics, such as nonlinear functional analysis, mathematical analysis, operator theory, and general topology. Historically, the study of fixed point theory has developed in two major branches: the first is fixed point theory for contraction or contraction type mappings on complete metric spaces and the second is fixed point theory for continuous operators on compact and convex subsets of a normed space. Recently, there has been a lot of activities in the first branch and several fundamental fixed point results have been extended and generalized by many authors in different directions. In this paper, we mention some important of them and give some new fixed point results. Also, we support our results by giving a lot of nontrivial examples. First, we give some notations, which will be used in this paper. Let $\psi:[0, \infty) \rightarrow[0, \infty)$ be a function. For convenience, we consider the following properties of this function:

$$
\begin{aligned}
& \left(\psi_{1}\right) \psi \text { is nondecreasing, } \\
& \left(\psi_{2}\right) \lim _{n \rightarrow \infty} \psi^{n}(t)=0 \text { for all } t \geq 0, \\
& \left(\psi_{3}\right) \psi(t)<t \text { for } t>0,
\end{aligned}
$$

$\left(\psi_{4}\right) \psi(0)=0$,

$\left(\psi_{5}\right) \psi$ is continuous,

$\left(\psi_{6}\right) \psi$ is upper semicontinuous from the right,

$\left(\psi_{7}\right) \sum_{n=1}^{\infty} \psi^{n}(t)<\infty$ for any $t>0$.

In the light of the above properties, the following hold: if $\psi_{1}$ and $\psi_{2}$ satisfied, then $\psi_{3}$ holds. If $\psi_{1}$ and $\psi_{3}$ are satisfied, then $\psi_{4}$ holds. If $\psi_{3}$ and $\psi_{5}$ are satisfied, then $\psi_{2}$ and $\psi_{4}$ hold.

Denoted by $\Psi$ the family of functions $\psi:[0, \infty) \rightarrow$ $[0, \infty)$ satisfying $\psi_{1}$ and $\psi_{2}$, which is called comparison functions in the literature (see [1]), by $\Phi$ the family of functions $\psi:[0, \infty) \rightarrow[0, \infty)$ satisfying $\psi_{1}$ and $\psi_{7}$, which is called (c)-comparison functions in the literature (see [1]), and by $\Upsilon$ the family of functions $\psi:[0, \infty) \rightarrow[0, \infty)$ satisfying $\psi_{3}$ and $\psi_{6}$. Now, we give some examples showing the relations between the sets $\Phi, \Psi$, and $\Upsilon$. First, it is clear that $\Phi \subset \Psi$.

Example 1. Let $\psi:[0, \infty) \rightarrow[0, \infty)$ be defined by $\psi(t)=\lambda t$, where $\lambda \in[0,1)$, and then $\psi \in \Phi \cap \Upsilon$.

Example 2. Let $\psi:[0, \infty) \rightarrow[0, \infty)$ be defined by $\psi(t)=$ $t /(1+t)$, and then $\psi \in \Psi \cap \Upsilon$, but $\psi \notin \Phi$. 
Example 3. Let $\psi:[0, \infty) \rightarrow[0, \infty)$ be defined by

$$
\psi(t)= \begin{cases}\frac{t}{3}, & 0 \leq t \leq \frac{2}{3} \\ \frac{t}{2}-\frac{1}{18}, & t>\frac{2}{3},\end{cases}
$$

and then $\psi \in \Phi$, but $\psi \notin \Upsilon$.

Example 4. Let $\psi:[0, \infty) \rightarrow[0, \infty)$ be defined by

$$
\psi(t)= \begin{cases}\frac{t}{1+t}, & 0 \leq t \leq 1 \\ \frac{2 t}{3}, & t>1,\end{cases}
$$

and then $\psi \in \Psi$, but $\psi \notin \Phi \cup \Upsilon$.

Example 5. Let $\psi:[0, \infty) \rightarrow[0, \infty)$ be defined by

$$
\psi(t)= \begin{cases}\frac{t}{1+t}, & 0 \leq t \leq 1 \\ \frac{1}{2 t}, & t>1,\end{cases}
$$

and then $\psi \in \Upsilon$, but $\psi \notin \Psi$.

In their recent paper, Samet et al. [2] introduced the notions of $\alpha$-admissible and $\alpha-\psi$-contractive mappings and then gave some fixed point results for such mappings. Their results are closely related to some ordered fixed point results. Then, using their idea, some authors presented fixed point results for single and multivalued mappings (see, e.g., [2-6]).

Definition 6 (see [2]). Let $(X, d)$ be a metric space, let $T$ be a self-map on $X, \psi \in \Phi$, and let $\alpha: X \times X \rightarrow[0, \infty)$ be a function. Then $T$ is called $\alpha-\psi$-contractive whenever

$$
\alpha(x, y) d(T x, T y) \leq \psi(d(x, y))
$$

for all $x, y \in X$.

Note that every Banach contraction mapping is an $\alpha-\psi$ contractive mapping with $\alpha(x, y)=1$ and $\psi(t)=\lambda t$ for some $\lambda \in[0,1)$.

Definition 7 (see [2]). $T$ is called $\alpha$-admissible whenever $\alpha(x, y) \geq 1$ implies $\alpha(T x, T y) \geq 1$.

There exist some examples for $\alpha$-admissible mappings in [2]. For convenience, we mention here one of them. Let $X=$ $[0, \infty)$. Define $T: X \rightarrow X$ and $\alpha: X \times X \rightarrow[0, \infty)$ by $T x=\sqrt{x}$ for all $x \in X$ and $\alpha(x, y)=e^{x-y}$ for $x \geq y$ and $\alpha(x, y)=0$ for $x<y$. Then $T$ is $\alpha$-admissible.

Theorem 8 (see [2]). Let $(X, d)$ be a complete metric space and let $T: X \rightarrow X$ be an $\alpha$-admissible and $\alpha-\psi$-contractive mapping. If there exists $x_{0} \in X$ such that $\alpha\left(x_{0}, T x_{0}\right) \geq 1$ and $T$ is continuous, then $T$ has a fixed point.

The following theorems are a generalization of Theorem 8 .
Theorem 9 (see [3]). Let $(X, d)$ be a complete metric space and let $T: X \rightarrow X$ be an $\alpha$-admissible mapping satisfying

$$
\alpha(x, y) d(T x, T y) \leq \psi(M(x, y))
$$

for all $x, y \in X$, where $\psi \in \Phi$ and

$$
\begin{gathered}
M(x, y) \\
=\max \{d(x, y), d(x, T x), d(y, T y), \\
\left.\frac{d(x, T y)+d(y, T x)}{2}\right\} .
\end{gathered}
$$

If there exists $x_{0} \in X$ such that $\alpha\left(x_{0}, T x_{0}\right) \geq 1$ and $T$ is continuous, then $T$ has a fixed point.

Theorem 10 (see $[4])$. Let $(X, d)$ be a complete metric space and let $T: X \rightarrow X$ be an $\alpha$-admissible mapping satisfying. Assume that

$$
\begin{aligned}
x, y \in X, \quad \alpha(x, y) & \geq 1 \Longrightarrow d(T x, T y) \\
& \leq \psi(m(x, y)),
\end{aligned}
$$

where $\psi \in \Phi$ and

$$
\begin{gathered}
m(x, y) \\
=\max \left\{d(x, y), \frac{d(x, T x)+d(y, T y)}{2},\right. \\
\left.\frac{d(x, T y)+d(y, T x)}{2}\right\} .
\end{gathered}
$$

If there exists $x_{0} \in X$ such that $\alpha\left(x_{0}, T x_{0}\right) \geq 1$ and $T$ is continuous or $X$ is regular, then $T$ has a fixed point.

For the sake of brevity, we will say that $X$ is regular whenever, for any sequence $\left\{x_{n}\right\}$ in $X$ with $\alpha\left(x_{n}, x_{n+1}\right) \geq 1$ for all $n \in \mathbb{N} \cup\{0\}$ and $x_{n} \rightarrow x$ as $n \rightarrow \infty$, we have $\alpha\left(x_{n}, x\right) \geq 1$ for all $n \in \mathbb{N} \cup\{0\}$.

The aim of this paper is to extend and generalize the above results. Note that, in these theorems, the function $\psi$ belongs to the class $\Phi$; that is, $\psi$ is (c)-comparison function. Also, in Theorem 10, the contractive condition is used with $m(x, y)$. In this paper, we give three existence results. In the first result, the contractive condition (7) will be generalized to almost contraction case. Here we take $\psi \in \Phi$, but we use $M(x, y)$ instead of $m(x, y)$. In the second result, we take $\psi$ in $\Psi$, which is a wider class of $\Phi$. And in the third result, we take $\psi$ in $\Upsilon$, which is a different class of $\Phi$. Also, we present some uniqueness theorems and some supporting examples.

\section{Existence Results}

Our first result is almost contraction version of fixed points of $\alpha$-admissible mapping. We can find detailed information about almost contractions in [7-12]. 
Theorem 11. Let $(X, d)$ be a complete metric space and let $T$ be an $\alpha$-admissible mapping. Assume that

$$
\begin{aligned}
\alpha(x, y) \geq 1 \Longrightarrow d(T x, T y) \leq & \psi(M(x, y)) \\
& +L \min \{d(y, T x), d(x, T y)\}
\end{aligned}
$$

holds for all $x, y \in X$, where $\psi \in \Phi, L \geq 0$, and $M(x, y)$ as in Theorem 9. Also, suppose $T$ is continuous or $X$ is regular and there exists $x_{0} \in X$ such that $\alpha\left(x_{0}, T x_{0}\right) \geq 1$. Then $T$ has a fixed point.

Proof. Let $x_{0} \in X$ such that $\alpha\left(x_{0}, T x_{0}\right) \geq 1$. Define a sequence $\left\{x_{n}\right\}$ in $X$ by

$$
x_{n}=T^{n} x_{0}=T x_{n-1}
$$

for all $n \in \mathbb{N}$. If $x_{n}=x_{n+1}$ for some $n \in \mathbb{N}$, then $x_{n}$ is a fixed point for $T$ and result is proved. So, we suppose that $x_{n} \neq x_{n+1}$ for all $n \in \mathbb{N}$. Since $T$ is $\alpha$-admissible mapping and $\alpha\left(x_{0}, T x_{0}\right) \geq 1$, we deduce that $\alpha\left(x_{1}, x_{2}\right)=\alpha\left(T x_{0}, T^{2} x_{0}\right)$. Continuing this process, we get $\alpha\left(x_{n}, x_{n+1}\right) \geq 1$ for all $n \in$ $\mathbb{N} \cup\{0\}$. Now by (9) with $x=x_{n}, y=x_{n+1}$, we get

$$
\begin{aligned}
d\left(x_{n+1}, x_{n+2}\right)= & d\left(T x_{n}, T x_{n+1}\right) \\
\leq & \psi\left(M\left(x_{n}, x_{n+1}\right)\right) \\
& +L \min \left\{d\left(x_{n+1}, T x_{n}\right), d\left(x_{n}, T x_{n+1}\right)\right\} \\
= & \psi\left(M\left(x_{n}, x_{n+1}\right)\right) \\
& +L \min \left\{d\left(x_{n+1}, x_{n+1}\right), d\left(x_{n}, T x_{n+1}\right)\right\} \\
= & \psi\left(M\left(x_{n}, x_{n+1}\right)\right),
\end{aligned}
$$

where

$$
\begin{aligned}
& M\left(x_{n}, x_{n+1}\right) \\
& =\max \left\{d\left(x_{n}, x_{n+1}\right), d\left(x_{n}, T x_{n}\right), d\left(x_{n+1}, T x_{n+1}\right),\right. \\
& \left.\quad \frac{d\left(x_{n}, T x_{n+1}\right)+d\left(x_{n+1}, T x_{n}\right)}{2}\right\} \\
& =\max \left\{d\left(x_{n}, x_{n+1}\right), d\left(x_{n+1}, x_{n+2}\right), \frac{d\left(x_{n}, x_{n+2}\right)}{2}\right\} \\
& =\max \left\{d\left(x_{n}, x_{n+1}\right), d\left(x_{n+1}, x_{n+2}\right)\right\} .
\end{aligned}
$$

If $d\left(x_{n+1}, x_{n+2}\right) \geq d\left(x_{n}, x_{n+1}\right)$ for some $n \in \mathbb{N} \cup\{0\}$, then from (11), we have

$$
\begin{aligned}
d\left(x_{n+1}, x_{n+2}\right) & \leq \psi\left(M\left(x_{n}, x_{n+1}\right)\right) \\
& =\psi\left(\max \left\{d\left(x_{n}, x_{n+1}\right), d\left(x_{n+1}, x_{n+2}\right)\right\}\right) \\
& =\psi\left(d\left(x_{n+1}, x_{n+2}\right)\right) \\
& <d\left(x_{n+1}, x_{n+2}\right),
\end{aligned}
$$

which is a contradiction. Thus $d\left(x_{n+1}, x_{n+2}\right)<d\left(x_{n}, x_{n+1}\right)$ for all $n \in \mathbb{N} \cup\{0\}$ and so from (11), we have

$$
d\left(x_{n+1}, x_{n+2}\right) \leq \psi\left(d\left(x_{n}, x_{n+1}\right)\right) .
$$

By induction, we have

$$
d\left(x_{n+1}, x_{n+2}\right) \leq \psi^{n+1}\left(d\left(x_{0}, x_{1}\right)\right)
$$

for all $n \in \mathbb{N} \cup\{0\}$. Now, for each $m, n \in \mathbb{N}, m>n$, we have

$$
d\left(x_{n}, x_{m}\right) \leq \sum_{k=n}^{m-1} d\left(x_{k}, x_{k+1}\right) \leq \sum_{k=n}^{m-1} \psi^{k}\left(d\left(x_{0}, x_{1}\right)\right) \text {. }
$$

Therefore, $\left\{x_{n}\right\}$ is a Cauchy sequence in $X$. Since $X$ is complete, there exists $z \in X$ such that $\lim _{n \rightarrow \infty} x_{n}=z$. If $T$ is continuous, then we have

$$
T z=\lim _{n \rightarrow \infty} T x_{n}=\lim _{n \rightarrow \infty} x_{n+1}=z .
$$

So, $z$ is a fixed point of $T$. Now, suppose $X$ is regular. Since

$$
\alpha\left(x_{n}, x_{n+1}\right) \geq 1
$$

for all $n \in \mathbb{N} \cup\{0\}$ and $x_{n} \rightarrow z$ as $n \rightarrow \infty$, then we have

$$
\alpha\left(x_{n}, z\right) \geq 1
$$

for all $n \in \mathbb{N} \cup\{0\}$. From (9) we have

$$
\begin{aligned}
d\left(x_{n+1}, T z\right)= & d\left(T x_{n}, T z\right) \\
\leq & \psi\left(M\left(x_{n}, z\right)\right) \\
& +L \min \left\{d\left(z, T x_{n}\right), d\left(x_{n}, T z\right)\right\} \\
= & \psi\left(M\left(x_{n}, z\right)\right) \\
& +L \min \left\{d\left(z, x_{n+1}\right), d\left(x_{n}, T z\right)\right\},
\end{aligned}
$$

where

$$
\begin{aligned}
M\left(x_{n}, z\right)=\max \left\{d\left(x_{n}, z\right), d\left(x_{n}, T x_{n}\right), d(z, T z),\right. & \left.\frac{1}{2}\left[d\left(x_{n}, T z\right)+d\left(z, T x_{n}\right)\right]\right\} \\
= & \max \left\{d\left(x_{n}, z\right), d\left(x_{n}, x_{n+1}\right), d(z, T z),\right. \\
& \left.\frac{1}{2}\left[d\left(x_{n}, T z\right)+d\left(z, x_{n+1}\right)\right]\right\} \\
& \max \left\{d\left(x_{n}, z\right), d\left(x_{n}, x_{n+1}\right), d(z, T z),\right. \\
& \left.\frac{1}{2}\left[d\left(x_{n}, z\right)+d(z, T z)+d\left(z, x_{n+1}\right)\right]\right\} .
\end{aligned}
$$

Now, suppose $d(z, T z)>0$. Taking into account (15) and $\lim _{n \rightarrow \infty} x_{n}=z$, there exists $n_{0} \in \mathbb{N}$ such that $d\left(x_{n}, z\right)<$ 
$d(z, T z) / 2$ and $d\left(x_{n}, x_{n+1}\right)<d(z, T z) / 2$ for all $n \geq n_{0}$. Therefore we have

$$
\begin{aligned}
M\left(x_{n}, z\right) \leq \max \{d & \left(x_{n}, z\right), d\left(x_{n}, x_{n+1}\right), d(z, T z) \\
& \left.\frac{1}{2}\left[d\left(x_{n}, z\right)+d(z, T z)+d\left(z, x_{n+1}\right)\right]\right\}
\end{aligned}
$$$$
\leq d(z, T z)
$$

for all $n \geq n_{0}$. Now, from (20), we obtain

$$
\begin{aligned}
d\left(x_{n+1}, T z\right) \leq & \psi(d(z, T z)) \\
& +L \min \left\{d\left(z, x_{n+1}\right), d\left(x_{n}, T z\right)\right\}
\end{aligned}
$$

for all $n \geq n_{0}$. Letting $n \rightarrow \infty$ in the last equality, we get that

$$
d(z, T z) \leq \psi(d(z, T z))<d(z, T z),
$$

which is a contradiction. Therefore $d(z, T z)=0$ and so $T$ has a fixed point.

Remark 12. In Theorem 11, if we take $L=0$, then we obtain an extension of Theorem 10. Even if $L=0$, we can extend Theorem 10 by taking the function $\psi$ from $\Psi$ and taking $M(x, y)$ instead of $m(x, y)$ as follows.

Theorem 13. Let $(X, d)$ be a complete metric space and let $T$ be an $\alpha$-admissible mapping. Assume that

$$
\alpha(x, y) \geq 1 \Longrightarrow d(T x, T y) \leq \psi(M(x, y))
$$

holds for all $x, y \in X$, where $\psi \in \Psi$ and $M(x, y)$ as in Theorem 11. Also, suppose $T$ is continuous or $X$ is regular and there exists $x_{0} \in X$ such that $\alpha\left(x_{0}, T x_{0}\right) \geq 1$. Then $T$ has a fixed point.

Proof. Let $x_{0} \in X$ such that $\alpha\left(x_{0}, T x_{0}\right) \geq 1$. As in the proof of Theorem 11, we can construct a sequence $\left\{x_{n}\right\}$ in $X$ and we can obtain (15) if the consecutive terms are different (otherwise, $T$ has a fixed point). Now we show that $\left\{x_{n}\right\}$ is a Cauchy sequence. Let $\varepsilon>0$. Taking into account (15), there exists $n_{0} \in \mathbb{N}$, such that $d\left(x_{n_{0}}, x_{n_{0}+1}\right)<\varepsilon-\psi(\varepsilon)$. Therefore we have

$$
\begin{aligned}
M\left(x_{n_{0}}, x_{n_{0}+1}\right)=\max \left\{d\left(x_{n_{0}}, x_{n_{0}+1}\right), d\left(x_{n_{0}}, T x_{n_{0}}\right),\right. \\
d\left(x_{n_{0}+1}, T x_{n_{0}+1}\right), \\
\frac{1}{2}\left[d\left(x_{n_{0}}, T x_{n_{0}+1}\right)\right. \\
\left.\left.+d\left(x_{n_{0}+1}, T x_{n_{0}}\right)\right]\right\} \\
=\max \left\{d\left(x_{n_{0}}, x_{n_{0}+1}\right), d\left(x_{n_{0}+1}, x_{n_{0}+2}\right)\right\} \\
<\max \{\varepsilon-\psi(\varepsilon), \psi(\varepsilon-\psi(\varepsilon))\} \\
=\varepsilon-\psi(\varepsilon)
\end{aligned}
$$

and so from (25)

$$
\begin{aligned}
d\left(x_{n_{0}}, x_{n_{0}+2}\right) & \leq d\left(x_{n_{0}}, x_{n_{0}+1}\right)+d\left(x_{n_{0}+1}, x_{n_{0}+2}\right) \\
& <\varepsilon-\psi(\varepsilon)+d\left(T x_{n_{0}}, T x_{n_{0}+1}\right) \\
& \leq \varepsilon-\psi(\varepsilon)+\psi\left(M\left(x_{n_{0}}, x_{n_{0}+1}\right)\right) \\
& \leq \varepsilon-\psi(\varepsilon)+\psi(\varepsilon-\psi(\varepsilon)) \\
& \leq \varepsilon-\psi(\varepsilon)+\psi(\varepsilon)=\varepsilon .
\end{aligned}
$$

Again using (25) we have

$$
\begin{aligned}
d\left(x_{n_{0}}, x_{n_{0}+3}\right) & \leq d\left(x_{n_{0}}, x_{n_{0}+1}\right)+d\left(x_{n_{0}+1}, x_{n_{0}+3}\right) \\
& <\varepsilon-\psi(\varepsilon)+d\left(T x_{n_{0}}, T x_{n_{0}+2}\right) \\
& \leq \varepsilon-\psi(\varepsilon)+\psi\left(M\left(x_{n_{0}}, x_{n_{0}+2}\right)\right),
\end{aligned}
$$

where

$$
\begin{aligned}
& M\left(x_{n_{0}}, x_{n_{0}+2}\right)=\max \left\{d\left(x_{n_{0}}, x_{n_{0}+2}\right), d\left(x_{n_{0}}, T x_{n_{0}}\right),\right. \\
& d\left(x_{n_{0}+2}, T x_{n_{0}+2}\right), \\
& \frac{1}{2}\left[d\left(x_{n_{0}}, T x_{n_{0}+2}\right)\right. \\
& \left.\left.+d\left(x_{n_{0}+2}, T x_{n_{0}}\right)\right]\right\} \\
& =\max \left\{d\left(x_{n_{0}}, x_{n_{0}+2}\right), d\left(x_{n_{0}}, x_{n_{0}+1}\right)\right. \text {, } \\
& d\left(x_{n_{0}+2}, x_{n_{0}+3}\right) \\
& \frac{1}{2}\left[d\left(x_{n_{0}}, x_{n_{0}+3}\right)\right. \\
& \left.\left.+d\left(x_{n_{0}+2}, x_{n_{0}+1}\right)\right]\right\} \\
& \leq \max \left\{\varepsilon, \varepsilon-\psi(\varepsilon), \psi^{2}(\varepsilon-\psi(\varepsilon))\right. \text {, } \\
& \frac{1}{2}\left[d\left(x_{n_{0}}, x_{n_{0}+3}\right)\right. \\
& +\psi(\varepsilon-\psi(\varepsilon))]\} \\
& \leq \max \left\{\varepsilon, \frac{1}{2}\left[d\left(x_{n_{0}}, x_{n_{0}+3}\right)+\psi(\varepsilon)\right]\right\} \text {. } \\
& \text { If } \varepsilon \leq(1 / 2)\left[d\left(x_{n_{0}}, x_{n_{0}+3}\right)+\psi(\varepsilon)\right] \text {, then, from (28), we have } \\
& \begin{aligned}
d\left(x_{n_{0}}, x_{n_{0}+3}\right) & <\varepsilon-\psi(\varepsilon)+\psi\left(\frac{1}{2}\left[d\left(x_{n_{0}}, x_{n_{0}+3}\right)+\psi(\varepsilon)\right]\right) \\
& <\varepsilon-\psi(\varepsilon)+\frac{1}{2}\left[d\left(x_{n_{0}}, x_{n_{0}+3}\right)+\psi(\varepsilon)\right]
\end{aligned}
\end{aligned}
$$


and so

$$
\frac{1}{2} d\left(x_{n_{0}}, x_{n_{0}+3}\right)<\varepsilon-\frac{\psi(\varepsilon)}{2}
$$

Therefore, we have

$$
\frac{1}{2}\left[d\left(x_{n_{0}}, x_{n_{0}+3}\right)+\psi(\varepsilon)\right]<\varepsilon,
$$

which is a contradiction. Thus, $\varepsilon>(1 / 2)\left[d\left(x_{n_{0}}, x_{n_{0}+3}\right)+\psi(\varepsilon)\right]$ and so, from (28), we have

$$
d\left(x_{n_{0}}, x_{n_{0}+3}\right)<\varepsilon
$$

By continuing this way, we can obtain

$$
d\left(x_{n_{0}}, x_{n_{0}+k}\right)<\varepsilon
$$

for all $k \in \mathbb{N}$. Now let $m, n \in \mathbb{N}$ with $m>n \geq n_{0}$, and then

$$
d\left(x_{m}, x_{n}\right) \leq d\left(x_{m}, x_{n_{0}}\right)+d\left(x_{n_{0}}, x_{n}\right)<2 \varepsilon ;
$$

that is, $\left\{x_{n}\right\}$ is a Cauchy sequence in $X$. The rest of the proof can be made as in the proof of Theorem 11 .

In the following theorem we take the function $\psi$ from $\Upsilon$ instead of $\Psi$.

Theorem 14. Let $(X, d)$ be a complete metric space and let $T$ be an $\alpha$-admissible mapping. Assume that

$$
\alpha(x, y) \geq 1 \Longrightarrow d(T x, T y) \leq \psi(M(x, y))
$$

holds for all $x, y \in X$, where $\psi \in \Upsilon$, and $M(x, y)$ as in Theorem 11. Also, suppose $T$ is continuous or $X$ is regular and there exists $x_{0} \in X$ such that $\alpha\left(x_{0}, T x_{0}\right) \geq 1$. Then $T$ has a fixed point.

Proof. Let $x_{0} \in X$ such that $\alpha\left(x_{0}, T x_{0}\right) \geq 1$. As in the proof of Theorem 11, we can construct a sequence $\left\{x_{n}\right\}$ in $X$ and we may assume that $x_{n} \neq x_{n+1}$ for all $n \in \mathbb{N}$. For the sake of brevity we put $d_{n}=d\left(x_{n}, x_{n+1}\right)$. Since $T$ is an $\alpha$-admissible mapping, we can obtain as in the proof of Theorem 11 that

$$
\begin{aligned}
d_{n+1} & =d\left(x_{n+1}, x_{n+2}\right) \\
& =d\left(T x_{n}, T x_{n+1}\right) \\
& \leq \psi\left(M\left(x_{n}, x_{n+1}\right)\right) \\
& =\psi\left(\max \left\{d_{n}, d_{n+1}\right\}\right) .
\end{aligned}
$$

Therefore, it should be $d_{n+1}<d_{n}$ for all integer $n \geq 0$ and so, from (37), we have

$$
d_{n+1} \leq \psi\left(d_{n}\right)<d_{n}
$$

Consequently, the sequence $\left\{d_{n}\right\}$ of positive numbers is decreasing and bounded below. So, there exists $\lambda \geq 0$ such that $\lim _{n \rightarrow \infty} d_{n}=\lambda$. We claim that $\lambda=0$. Suppose to the contrary that $\lambda>0$. Using the fact that $\psi$ is upper semicontinuous from the right function, we get from (38)

$$
\lambda=\limsup _{n \rightarrow \infty} d_{n+1} \leq \limsup _{n \rightarrow \infty} \psi\left(d_{n}\right) \leq \psi(\lambda)<\lambda,
$$

which is a contradiction. Hence, we conclude that $\lambda=0$; that is,

$$
\lim _{n \rightarrow \infty} d_{n}=\lim _{n \rightarrow \infty} d\left(x_{n}, x_{n+1}\right)=0 .
$$

Now, we prove that the sequence $\left\{x_{n}\right\}$ is Cauchy in $X$. Suppose, to the contrary, there exists $\varepsilon>0$ such that

$$
d\left(x_{m_{k}}, x_{n_{k}}\right) \geq \varepsilon
$$

where $\left\{x_{m_{k}}\right\}$ and $\left\{x_{n_{k}}\right\}$ are subsequences of $\left\{x_{n}\right\}$ with $m_{k}>$ $n_{k} \geq k$ for all $k \in \mathbb{N}$. Moreover, $m_{k}$ is chosen as the smallest integer satisfying (41). Thus, we have

$$
d\left(x_{m_{k}-1}, x_{n_{k}}\right)<\varepsilon .
$$

By the triangle inequality, we get

$$
\begin{aligned}
\varepsilon & \leq d\left(x_{m_{k}}, x_{n_{k}}\right) \\
& \leq d\left(x_{m_{k}}, x_{m_{k}-1}\right)+d\left(x_{m_{k}-1}, x_{n_{k}}\right)<d_{m_{k}}+\varepsilon .
\end{aligned}
$$

Letting $k \rightarrow \infty$ in above inequality and using (40), we get that

$$
\lim _{k \rightarrow \infty} d\left(x_{m_{k}}, x_{n_{k}}\right)=\varepsilon
$$

Now let $k_{0} \in \mathbb{N}$ be such that $d_{n_{k}}<\varepsilon$ and $d_{m_{k}}<\varepsilon$ for all $k \geq k_{0}$. Then

$$
\begin{aligned}
d\left(x_{m_{k}}, x_{n_{k}}\right) & \leq M\left(x_{m_{k}}, x_{n_{k}}\right) \\
& \leq d\left(x_{m_{k}}, x_{n_{k}}\right)+\frac{1}{2}\left(d_{n_{k}}+d_{m_{k}}\right)
\end{aligned}
$$

for all $k \geq k_{0}$. Using (40) and (44) and letting $k \rightarrow \infty$ in (45), we get

$$
\lim _{k \rightarrow \infty} M\left(x_{m_{k}}, x_{n_{k}}\right)=\varepsilon
$$

Since $M\left(x_{m_{k}}, x_{n_{k}}\right) \geq \varepsilon$ for all $k \in \mathbb{N}$ and $\psi$ is upper semicontinuous from the right function, we deduce that

$$
\limsup _{n \rightarrow \infty} \psi\left(M\left(x_{m_{k}}, x_{n_{k}}\right)\right) \leq \psi(\varepsilon) .
$$

On the other hand, for each $k \in \mathbb{N}$, we have

$$
\begin{aligned}
\varepsilon \leq & d\left(x_{m_{k}}, x_{n_{k}}\right) \\
\leq & d\left(x_{m_{k}}, x_{m_{k}+1}\right)+d\left(x_{m_{k}+1}, x_{n_{k}+1}\right) \\
& +d\left(x_{n_{k}+1}, x_{n_{k}}\right) \\
= & d_{m_{k}}+d\left(T x_{m_{k}}, T x_{n_{k}}\right)+d_{n_{k}} \\
\leq & d_{m_{k}}+\psi\left(M\left(x_{m_{k}}, x_{n_{k}}\right)\right)+d_{n_{k}},
\end{aligned}
$$


so

$$
\varepsilon \leq \limsup _{n \rightarrow \infty} \psi\left(M\left(x_{m_{k}}, x_{n_{k}}\right)\right) \leq \psi(\varepsilon)<\varepsilon,
$$

which is a contradiction. Thus $\left\{x_{n}\right\}$ is a Cauchy sequence in $X$. Since $X$ is complete, there exists $z \in X$ such that $\lim _{n \rightarrow \infty} x_{n}=$ $z$. If $T$ is continuous, then we have

$$
T z=\lim _{n \rightarrow \infty} T x_{n}=\lim _{n \rightarrow \infty} x_{n+1}=z .
$$

So $z$ is a fixed point of $T$. Now, suppose $X$ is regular and $d(z, T z)>0$. We first note that

$$
\begin{gathered}
d(z, T z) \leq M\left(x_{n}, z\right), \\
\lim _{n \rightarrow \infty} M\left(x_{n}, z\right)=d(z, T z) ;
\end{gathered}
$$

hence by the upper semicontinuity of $\psi$, we get

$$
\limsup _{n \rightarrow \infty} \psi\left(M\left(x_{n}, z\right)\right) \leq \psi(d(z, T z)) .
$$

On the other hand, since $X$ is regular,

$$
\alpha\left(x_{n}, x_{n+1}\right) \geq 1
$$

for all integer $n \geq 0$ and $x_{n} \rightarrow z$ as $n \rightarrow \infty$, then we have

$$
\alpha\left(x_{n}, z\right) \geq 1
$$

for all integer $n \geq 0$. Thus, from (36), we have

$$
d\left(x_{n+1}, T z\right)=d\left(T x_{n}, T z\right) \leq \psi\left(M\left(x_{n}, z\right)\right),
$$

and taking limit supremum, we get

$$
\begin{aligned}
d(z, T z) & \leq \limsup _{n \rightarrow \infty} \psi\left(M\left(x_{n_{-1}}, z\right)\right) \\
& \leq \psi(d(z, T z)) \\
& <d(z, T z)
\end{aligned}
$$

which is a contradiction. Therefore $d(z, T z)=0$ and so $T$ has a fixed point.

\section{Uniqueness Results}

In this section, we consider some properties to obtain the uniqueness of the fixed point in the above theorems. For this, we denote the set of fixed points of $T$ by $\operatorname{Fix}(T)$.

Theorem 15. Assume that all hypotheses of Theorem 13 hold. Also suppose

$\forall x, y \in F i x(T)$, there exists $u \in X$ such that

$$
\alpha(x, u) \geq 1, \alpha(y, u) \geq 1,
$$

holds, then the fixed point of $T$ is unique.

Proof. Suppose $z$ and $w$ are two fixed points of $T$, and then there exists $u \in X$ such that $\alpha(z, u) \geq 1$ and $\alpha(w, u) \geq 1$. Since $T$ is an $\alpha$-admissible mapping, then

$$
\alpha\left(z, T^{n} u\right) \geq 1, \quad \alpha\left(w, T^{n} u\right) \geq 1
$$

for all $n \in \mathbb{N}$. Therefore

$$
\begin{aligned}
d\left(z, T^{n+1} u\right) & =d\left(T z, T^{n+1} u\right) \\
& \leq \psi\left(M\left(z, T^{n} u\right)\right) \\
& \leq \psi\left(\max \left\{d\left(z, T^{n} u\right), d\left(z, T^{n+1} u\right)\right\}\right)
\end{aligned}
$$

Without loss of generality, we can assume $d\left(z, T^{n} u\right)>0$ for all $n$. Therefore from (59) we have (note it should be $d\left(z, T^{n} u\right)>$ $d\left(z, T^{n+1} u\right)$. Otherwise we obtain a contradiction from (59))

$$
d\left(z, T^{n+1} u\right) \leq \psi\left(d\left(z, T^{n} u\right)\right) \leq \cdots \leq \psi^{n}(d(z, u)) .
$$

Letting $n \rightarrow \infty$ in the above inequality, we have $T^{n} u \rightarrow z$. Similarly, we can obtain $T^{n} u \rightarrow w$ and so $z=w$.

Remark 16. The condition (57) is not sufficient to obtain the uniqueness of the fixed point in Theorem 11.

Example 17. Let $X=[0,1]$ with the usual metric. Define $T$ : $X \rightarrow X$ and $\alpha: X \times X \rightarrow[0, \infty)$ by

$$
T x= \begin{cases}x^{2}, & x \in\left[0, \frac{1}{4}\right) \\ 0, & x \in\left[\frac{1}{4}, \frac{1}{3}\right) \\ 1, & x \in\left[\frac{1}{3}, 1\right],\end{cases}
$$

and $\alpha(x, y)=1$. Then, it is clear that $T$ is $\alpha$-admissible mapping and $X$ is regular. Also, there exists $x_{0} \in X$ such that $\alpha\left(x_{0}, T x_{0}\right) \geq 1$. Now, we show that (9) is satisfied with $\psi(t)=$ $t / 2$ and $L=10$. For this, we consider the following cases.

Case 1. If $x, y \in[0,1 / 4)$, then

$$
\begin{aligned}
d(T x, T y) & =\left|x^{2}-y^{2}\right| \\
& \leq \frac{1}{2}|x-y| \\
& \leq \psi(M(x, y))+L \min \{d(y, T x), d(x, T y)\} .
\end{aligned}
$$

Case 2. If $x, y \in[1 / 4,1 / 3)$, then $d(T x, T y)=0$.

Case 3. If $x, y \in[1 / 3,1]$, then $d(T x, T y)=0$.

Case 4. If $x \in[0,1 / 4)$ and $y \in[1 / 4,1 / 3)$, then

$$
\begin{aligned}
d(T x, T y) & =x^{2} \\
& \leq \min \left\{x, y-x^{2}\right\} \\
& \leq \psi(M(x, y))+L \min \{d(y, T x), d(x, T y)\} .
\end{aligned}
$$


Case 5. If $x \in[0,1 / 4)$ and $y \in[1 / 3,1]$, then

$$
\begin{aligned}
d(T x, T y) & =1-x^{2} \\
& \leq \frac{1}{2}(y-x)+10 \min \left\{1-x, y-x^{2}\right\} \\
& \leq \psi(M(x, y))+L \min \{d(y, T x), d(x, T y)\} .
\end{aligned}
$$

Case 6. If $x \in[1 / 4,1 / 3)$ and $y \in[1 / 3,1]$, then

$$
\begin{aligned}
d(T x, T y) & =1 \\
& \leq \frac{1}{2}(y-x)+10 \min \{1-x, y\} \\
& \leq \psi(M(x, y))+L \min \{d(y, T x), d(x, T y)\} .
\end{aligned}
$$

Therefore, all conditions of Theorem 11 are satisfied and so $T$ has a fixed point. Although the condition (57) of Theorem 15 is satisfied, the fixed point of $T$ is not unique.

In the following, we give a uniqueness theorem by adding some conditions in Theorem 11.

Theorem 18. Assume that all hypotheses of Theorem 11 hold. Also suppose for all $x, y \in F i x(T)$, there exists $u \in X$ such that $\alpha(x, u) \geq 1$ and $\alpha(y, u) \geq 1$ and

$$
\begin{aligned}
\alpha(x, y) & \geq 1 \Longrightarrow d(T x, T y) \\
& \leq \psi_{1}(M(x, y))+L_{1} \min \{d(x, T x), d(y, T y)\}
\end{aligned}
$$

holds for all $x, y \in X$, where $\psi_{1} \in \Psi$ and $L_{1} \geq 0$, then the fixed point of $T$ is unique.

Proof. Suppose $z$ and $w$ are two fixed points of $T$, and then there exists $u \in X$ such that $\alpha(z, u) \geq 1$ and $\alpha(w, u) \geq 1$. Since $T$ is an $\alpha$-admissible mapping, then

$$
\alpha\left(z, T^{n} u\right) \geq 1, \quad \alpha\left(w, T^{n} u\right) \geq 1
$$

for all $n \in \mathbb{N}$. Therefore from (66)

$$
\begin{aligned}
d\left(z, T^{n+1} u\right)= & d\left(T z, T^{n+1} u\right) \\
\leq & \psi_{1}\left(M\left(z, T^{n} u\right)\right) \\
& +L_{1} \min \left\{d(z, T z), d\left(T^{n} u, T^{n+1} u\right)\right\} \\
\leq & \psi_{1}\left(\max \left\{d\left(z, T^{n} u\right), d\left(z, T^{n+1} u\right)\right\}\right) .
\end{aligned}
$$

Without loss of generality, we can assume $d\left(z, T^{n} u\right)>0$ for all $n$. Therefore we have

$$
d\left(z, T^{n+1} u\right) \leq \psi_{1}\left(d\left(z, T^{n} u\right)\right) \leq \cdots \leq \psi_{1}^{n}(d(z, u)) .
$$

Letting $n \rightarrow \infty$ in the above inequality, we have $T^{n} u \rightarrow z$. Similarly, we can obtain $T^{n} u \rightarrow w$ and so $z=w$.

\section{Some Corollaries and Example}

Corollary 19. Let $(X, d)$ be a complete metric space and $T$ : $X \rightarrow X$ be $\alpha$-admissible. Assume that

$$
\begin{aligned}
\alpha(x, y) & \geq 1 \Longrightarrow d(T x, T y) \\
& \leq \psi(d(x, y))+L \min \{d(y, T x), d(x, T y)\}
\end{aligned}
$$

holds for all $x, y \in X$, where $\psi \in \Phi$ and $L \geq 0$. Also, suppose $T$ is continuous and there exists $x_{0} \in X$ such that $\alpha\left(x_{0}, T x_{0}\right) \geq 1$. Then $T$ has a fixed point.

Corollary 20. Let $(X, d)$ be a complete metric space and let $T$ be self-map of $X$. Assume that

$$
d(T x, T y) \leq \psi(M(x, y))+L \min \{d(y, T x), d(x, T y)\}
$$

holds for all $x, y \in X$, where $\psi \in \Phi, L \geq 0$, and $M(x, y)$ as in Theorem 9. Then $T$ has a fixed point.

Corollary 21. Let $(X, d)$ be a complete metric space and let $T$ be self-map of $X$. Assume that

$$
d(T x, T y) \leq \psi(d(x, y))+L \min \{d(y, T x), d(x, T y)\}
$$

holds for all $x, y \in X$, where $\psi \in \Phi$ and $L \geq 0$. Then $T$ has a fixed point.

Corollary 22. Let $(X, d)$ be a complete metric space and let $T: X \rightarrow X$ be $\alpha$-admissible. Assume that

$$
\alpha(x, y) \geq 1 \Longrightarrow d(T x, T y) \leq \psi(d(x, y))
$$

holds for all $x, y \in X$, where $\psi \in \Psi$ (or $\psi \in \Upsilon$ ). Also, suppose $T$ is continuous and there exists $x_{0} \in X$ such that $\alpha\left(x_{0}, T x_{0}\right) \geq 1$. Then $T$ has a fixed point.

Corollary 23. Let $(X, d)$ be a complete metric space and let $T$ be a self-map of $X$. Assume that

$$
d(T x, T y) \leq \psi(M(x, y))
$$

holds for all $x, y \in X$, where $\psi \in \Psi$ (or $\psi \in \Upsilon$ ), and $M(x, y)$ as in Theorem 9. Then $T$ has a unique fixed point.

Corollary 24. Let $(X, d)$ be a complete metric space and let $T$ be a self-map of $X$. Assume that

$$
d(T x, T y) \leq \psi(d(x, y))
$$

holds for all $x, y \in X$, where $\psi \in \Psi$ (or $\psi \in \Upsilon$ ). Then $T$ has a unique fixed point.

Example 25. Let $X=[0,1]$ with the usual metric. Define $T$ : $X \rightarrow X$ and $\alpha: X \times X \rightarrow[0, \infty)$ by

$$
\begin{gathered}
T x= \begin{cases}x^{2}, & x \in\left[0, \frac{1}{4}\right] \\
\frac{5 x-1}{4}, & x \in\left(\frac{1}{4}, 1\right],\end{cases} \\
\alpha(x, y)= \begin{cases}1, & x, y \in\left[0, \frac{1}{4}\right] \cup\{1\} \\
0, & \text { otherwise }\end{cases}
\end{gathered}
$$


for all $x, y \in X$. Then, it is clear that $T$ is $\alpha$-admissible mapping and $T$ is continuous. Also, $\alpha(1, T 1) \geq 1$. But, although $\alpha(1,1 / 4) \geq 1$, we cannot find any $\psi \in \Phi$ satisfying

$$
d\left(T 1, T \frac{1}{4}\right)=\frac{15}{16} \leq \psi\left(\frac{27}{32}\right)=\psi(m(x, y))=\psi(M(x, y)) .
$$

Therefore, Theorems 10 and 9 cannot be applied to this example. Now, we show that (9) is satisfied with $\psi(t)=t / 2$ and $L=4 / 3$. Let $\alpha(x, y) \geq 1$, and then $x, y \in[0,1 / 4] \cup\{1\}$. We have to consider the following cases.

Case 1. If $x, y \in[0,1 / 4]$, then

$$
\begin{aligned}
d(T x, T y) & =\left|x^{2}-y^{2}\right| \leq \frac{1}{2}|x-y| \\
& \leq \psi(M(x, y))+L \min \{d(x, T y), d(y, T x)\} .
\end{aligned}
$$

Case 2. If $x \in[0,1 / 4]$ and $y=1$, then

$$
\begin{aligned}
d(T x, T y) & =1-x^{2} \leq \frac{1}{2}(1-x)+1 \\
& \leq \psi(M(x, y))+L \min \{d(x, T y), d(y, T x)\} .
\end{aligned}
$$

Therefore, all conditions of Theorem 11 are satisfied and so $T$ has a fixed point in $X$.

\section{Applications}

In this section, we apply Corollary 22 to the following fourth order two-point boundary value problem:

$$
\begin{aligned}
& y^{(4)}(t)=f(t, y(t)), \quad t \in[0,1], \\
& y(0)=y(1)=y^{\prime}(0)=y^{\prime}(1)=0,
\end{aligned}
$$

which describes the bending of an elastic beam clamped at both endpoints. In [13], using an ordered version of Geraghty's fixed point result, an existence theorem for a nonnegative solution of (80) is given. The boundary value problem can be written as the integral equation (see [14])

$$
y(t)=\int_{0}^{1} G(t, s) f(s, y(s)) d s \quad \text { for } t \in[0,1],
$$

where $G(t, s)$ is the Green's function given by

$$
G(t, s)=\frac{1}{6} \begin{cases}t^{2}(1-s)^{2}[(s-t)+2(1-t) s], & 0 \leq t \leq s \leq 1 \\ s^{2}(1-t)^{2}[(t-s)+2(1-s) t], & 0 \leq s \leq t \leq 1\end{cases}
$$

Then it is clear that, $G(t, s)$ is continuous on $[0,1] \times[0,1]$, $G(0, s)=G(1, s)=0$, and $G(t, s) \geq 0$ for $t, s \in[0,1]$ and $\sup _{t \in[0,1]} \int_{0}^{1} G(t, s) d s=1 / 384$.

Here we will consider $C[0,1]$ with the uniform metric, that is, for $x, y \in C[0,1] d_{\infty}(x, y)=\sup _{t \in[0,1]}|x(t)-y(t)|$.
Then $\left(C[0,1], d_{\infty}\right)$ is a Banach space. For the convenience we consider the operator $T$ defined on $C[0,1]$ by

$$
T x(t)=\int_{0}^{1} G(t, s) f(s, x(s)) d s \quad \text { for } t \in[0,1] .
$$

Theorem 26. Under the following assumptions, Problem (80) has a solution.

(A) $f:[0,1] \times \mathbb{R} \rightarrow \mathbb{R}$ is bounded,

(B) there exist $\psi \in \Psi$ and $\theta: C[0,1] \times C[0,1] \rightarrow \mathbb{R}$ such that if $\theta(x, y) \geq 0$ for $x, y \in C[0,1]$, then for $s \in[0,1]$ we have

$$
0 \leq f(s, x(s))-f(s, y(s)) \leq 384 \psi(|x(s)-y(s)|),
$$

(C) if $\theta(x, y) \geq 0$, then $\theta(T x, T y) \geq 0$,

(D) there exists $x_{0} \in C[0,1]$ such that $\theta\left(x_{0}, T x_{0}\right) \geq 0$,

(E) if $\left\{x_{n}\right\}$ is a sequence in $C[0,1]$ such that $\theta\left(x_{n}, x_{n+1}\right) \geq 0$ for all $n \in \mathbb{N} \cup\{0\}$ and $x_{n} \rightarrow x$, then $\theta\left(x_{n}, x\right) \geq 0$ for all $n \in \mathbb{N} \cup\{0\}$.

Proof. Let $X=C[0,1]$ with the metric $d_{\infty}$. First, we show that $T: X \rightarrow X$. Let $x \in X$ and $t, t^{\prime} \in[0,1]$, and then, by the continuity of $G$ and the boundedness of $f$, we have

$$
\begin{aligned}
& \left|T x(t)-T x\left(t^{\prime}\right)\right| \\
& \quad=\left|\int_{0}^{1} G(t, s) f(s, x(s)) d s-\int_{0}^{1} G\left(t^{\prime}, s\right) f(s, x(s)) d s\right| \\
& \quad \leq \int_{0}^{1}\left|G(t, s)-G\left(t^{\prime}, s\right)\right||f(s, x(s))| d s \\
& \leq \sup _{s \in[0,1]}\left|G(t, s)-G\left(t^{\prime}, s\right)\right| \int_{0}^{1}|f(s, x(s))| d s \\
& \quad \leq M \sup _{s \in[0,1]}\left|G(t, s)-G\left(t^{\prime}, s\right)\right| \longrightarrow 0 \text { for }\left|t-t^{\prime}\right| \longrightarrow 0
\end{aligned}
$$

where $M>0$ such that $\sup _{s \in[0,1]}|f(s, x(s))| \leq M$.

Now, define $\alpha: X \times X \rightarrow[0, \infty)$ by

$$
\alpha(x, y)= \begin{cases}1, & \theta(x, y) \geq 0 \\ 0, & \text { otherwise }\end{cases}
$$


Then, it is clear that $T$ is $\alpha$-admissible. Also, for all $x, y \in X$ with $\alpha(x, y) \geq 1$, we have

$$
\begin{aligned}
& |T x(t)-T y(t)| \\
& \quad=\left|\int_{0}^{1} G(t, s) f(s, x(s)) d s-\int_{0}^{1} G(t, s) f(s, y(s)) d s\right| \\
& \quad \leq \int_{0}^{1} G(t, s)|f(s, x(s))-f(s, y(s))| d s \\
& \quad \leq \int_{0}^{1} G(t, s) 384 \psi(|x(s)-y(s)|) d s \\
& \quad \leq 384 \psi(d,(x, y)) \int_{0}^{1} G(t, s) d s \\
& \quad \leq \psi\left(d_{\infty}(x, y)\right)
\end{aligned}
$$

for all $t \in[0,1]$. Therefore

$$
\alpha(x, y) \geq 1 \Longrightarrow d_{\infty}(T x, T y) \leq \psi\left(d_{\infty}(x, y)\right)
$$

hold. Finally, by the condition (E), $X$ is regular. Therefore all hypotheses of Corollary 22 are satisfied and so $T$ has a fixed point in $X$. Thus, the problem $(80)$ has a solution in $C[0,1]$.

In the following, we also give an existence and uniqueness theorem for (80) under slight different conditions.

Theorem 27. Under the following assumptions, problem (80) has a unique solution.

(F) $f:[0,1] \times \mathbb{R} \rightarrow \mathbb{R}$ is bounded, nondecreasing with respect to second variable and $f(t, 0) \geq 0$ for all $t \in$ $[0,1]$,

(G) there exists $\psi \in \Psi$ such that, for $x, y \in C[0,1]$ with $x \preceq y$ and $s \in[0,1]$, we have

$$
f(s, y(s))-f(s, x(s)) \leq 384 \psi(|x(s)-y(s)|),
$$

where $x \leq y \Leftrightarrow x(t) \leq y(t)$ for all $t \in[0,1]$.

Proof. As in the proof of Theorem 26, we can show that $T$ : $X \rightarrow X$. Now define $\alpha: X \times X \rightarrow[0, \infty)$ by

$$
\alpha(x, y)= \begin{cases}1, & x \preceq y \\ 0, & \text { otherwise }\end{cases}
$$

Therefore, if $\alpha(x, y) \geq 1$, then $x \preceq y$ and $x(t) \leq y(t)$ for all $t \in[0,1]$. Since $f$ is nondecreasing with respect to second variable, we have

$$
\begin{aligned}
T x(t) & =\int_{0}^{1} G(t, s) f(s, x(s)) d s \\
& \leq \int_{0}^{1} G(t, s) f(s, y(s)) d s \\
& =T y(t)
\end{aligned}
$$

for all $t \in[0,1]$. Thus, we have $T x \preceq T y$ and so $\alpha(T x, T y) \geq 1$. It shows that $T$ is $\alpha$-admissible. Also, for all $x, y \in X$ with $\alpha(x, y) \geq 1$, we have

$$
\begin{aligned}
\mid T x & (t)-T y(t) \mid \\
& =\left|\int_{0}^{1} G(t, s) f(s, x(s)) d s-\int_{0}^{1} G(t, s) f(s, y(s)) d s\right| \\
& \leq \int_{0}^{1} G(t, s)|f(s, x(s))-f(s, y(s))| d s \\
& =\int_{0}^{1} G(t, s)[f(s, y(s))-f(s, x(s))] d s \\
& \leq \int_{0}^{1} G(t, s) 384 \psi(|x(s)-y(s)|) d s \\
& \leq 384 \psi(d \infty(x, y)) \int_{0}^{1} G(t, s) d s \\
& \leq \psi\left(d_{\infty}(x, y)\right)
\end{aligned}
$$

for all $t \in[0,1]$. Therefore

$$
\alpha(x, y) \geq 1 \Longrightarrow d_{\infty}(T x, T y) \leq \psi\left(d_{\infty}(x, y)\right)
$$

hold. Also, since $f(s, 0) \geq 0$, we have $0 \leq \int_{0}^{1} G(t, s) f(s, 0) d s=$ $T 0$ and so $\alpha(0, T 0) \geq 1$. Now, let $\alpha\left(x_{n}, x_{n+1}\right) \geq 1$ for all $n \in$ $\mathbb{N} \cup\{0\}$ and $x_{n} \rightarrow x$ in $X$. Then, we have $x_{n} \preceq x_{n+1}$ and so $x_{n}(t) \leq x_{n+1}(t)$ for all $t \in[0,1]$. Therefore, $x_{n}(t) \leq x(t)$ for all $t \in[0,1]$ and $n \in \mathbb{N} \cup\{0\}$. That is, $x_{n} \preceq x$ for all $n \in \mathbb{N} \cup\{0\}$ and so $\alpha\left(x_{n}, x\right) \geq 1$. Hence $X$ is regular. Therefore all existence hypotheses of Corollary 22 are satisfied and so $T$ has a fixed point in $X$. Thus, the problem (80) has a solution in $C[0,1]$. Finally, let $x$ and $y$ be two solutions of $(80)$, and then $u=\max \{x, y\} \in C[0,1]$ and $x \preceq u$ and $y \preceq u$. Thus, $\alpha(x, u) \geq 1$ and $\alpha(y, u) \geq 1$. Therefore, by Corollary 22 the solution is unique.

Example 28. Consider the nonlinear fourth order two point boundary value problem

$$
\begin{gathered}
y^{(4)}(t)=\gamma t+\left(1+t^{2}\right) \arctan (\beta y(t)), \quad t \in[0,1], \\
y(0)=y(1)=y^{\prime}(0)=y^{\prime}(1)=0,
\end{gathered}
$$

where $\gamma \geq 0,0 \leq \beta \leq 191$. In this case, $f(t, y)=\gamma t+(1+$ $\left.t^{2}\right) \operatorname{arc} \tan (\beta y)$. It is easy to see that $f:[0,1] \times \mathbb{R} \rightarrow \mathbb{R}$ is bounded and nondecreasing with respect to the second variable. Also, $f(t, 0)=\gamma t \geq 0$ for all $t \in[0,1]$. Now let $x, y \in C[0,1]$ with $x \preceq y$ then, for all $s \in[0,1]$,

$$
\begin{aligned}
& f(s, y(s))-f(s, x(s)) \\
& \quad=\left(1+s^{2}\right)[\arctan (\beta y(s))-\arctan (\beta x(s))]
\end{aligned}
$$




$$
\begin{aligned}
& \leq\left(1+s^{2}\right) \arctan (\beta[y(s)-x(s)]) \\
& \leq\left(1+s^{2}\right) \beta[y(s)-x(s)] \\
& \leq 2 \beta[y(s)-x(s)] \\
& \leq 382[y(s)-x(s)] \\
& =384 \psi(|y(s)-x(s)|),
\end{aligned}
$$

where $\psi(t)=191 t / 192$. Therefore, the conditions (F) and (G) of Theorem 27 are satisfied and so the boundary value problem (94) has a unique solution.

\section{Conflict of Interests}

The authors declare that there is no conflict of interests regarding the publication of this paper.

\section{References}

[1] V. Berinde, Iterative Approximation of Fixed Points, Springer, Berlin, Germany, 2007.

[2] B. Samet, C. Vetro, and P. Vetro, "Fixed point theorems for $\alpha-\psi$-contractive type mappings," Nonlinear Analysis. Theory, Methods \& Applications, vol. 75, no. 4, pp. 2154-2165, 2012.

[3] J. H. Asl, S. Rezapour, and N. Shahzad, "On fixed points of $\alpha-\psi$-contractive multifunctions," Fixed Point Theory and Applications, vol. 2012, article 212, p. 6, 2012.

[4] E. Karapinar and B. Samet, "Generalized $\alpha$ - $\psi$-contractive type mappings and related fixed point theorems with applications," Abstract and Applied Analysis, vol. 2012, Article ID 793486, 17 pages, 2012.

[5] B. Mohammadi, S. Rezapour, and N. Shahzad, "Some results on fixed points of $\alpha$ - $\psi$-Ciric generalized multifunctions," Fixed Point Theory and Applications, vol. 2013, article 24, 10 pages, 2013.

[6] N. Hussain, E. Karapınar, P. Salimi, and F. Akbar, " $\alpha$-admissible mappings and related fixed point theorems," Journal of Inequalities and Applications, vol. 2013, article 114, 11 pages, 2013.

[7] V. Berinde, "Approximating fixed points of weak $\varphi$-contractions using the Picard iteration," Fixed Point Theory, vol. 4, no. 2, pp. 131-147, 2003.

[8] V. Berinde, "On the approximation of fixed points of weak contractive mappings," Carpathian Journal of Mathematics, vol. 19, no. 1, pp. 7-22, 2003.

[9] V. Berinde, "General constructive fixed point theorems for Cirić-type almost contractions in metric spaces," Carpathian Journal of Mathematics, vol. 24, no. 2, pp. 10-19, 2008.

[10] V. Berinde, "Some remarks on a fixed point theorem for Ćirićtype almost contractions," Carpathian Journal of Mathematics, vol. 25, no. 2, pp. 157-162, 2009.

[11] M. Pacurar, "Sequences of almost contractions and fixed points," Carpathian Journal of Mathematics, vol. 24, no. 2, pp. 101-109, 2008.

[12] M. Pacurar, "Remark regarding two classes of almost contractions with unique fixed point," Creative Mathematics and Informatics, vol. 19, no. 2, pp. 178-183, 2010.

[13] J. Caballero, J. Harjani, and K. Sadarangani, "Uniqueness of positive solutions for a class of fourth-order boundary value pro-blems," Abstract and Applied Analysis, vol. 2011, Article ID 543035, 13 pages, 2011.

[14] M. Pei and S. K. Chang, "Monotone iterative technique and symmetric positive solutions for a fourth-order boundary value problem," Mathematical and Computer Modelling, vol. 51, no. 910, pp. 1260-1267, 2010. 


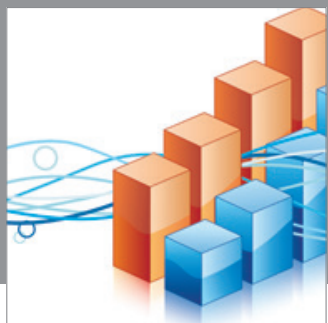

Advances in

Operations Research

mansans

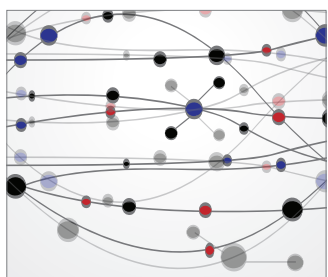

The Scientific World Journal
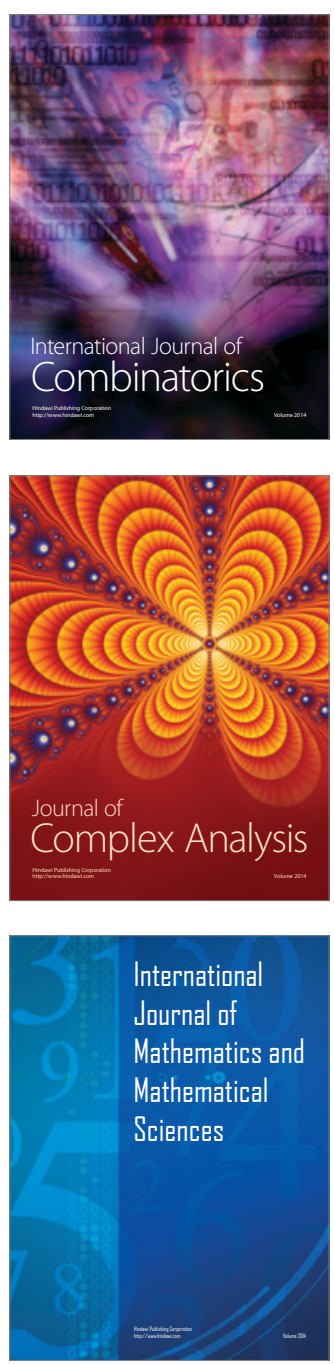
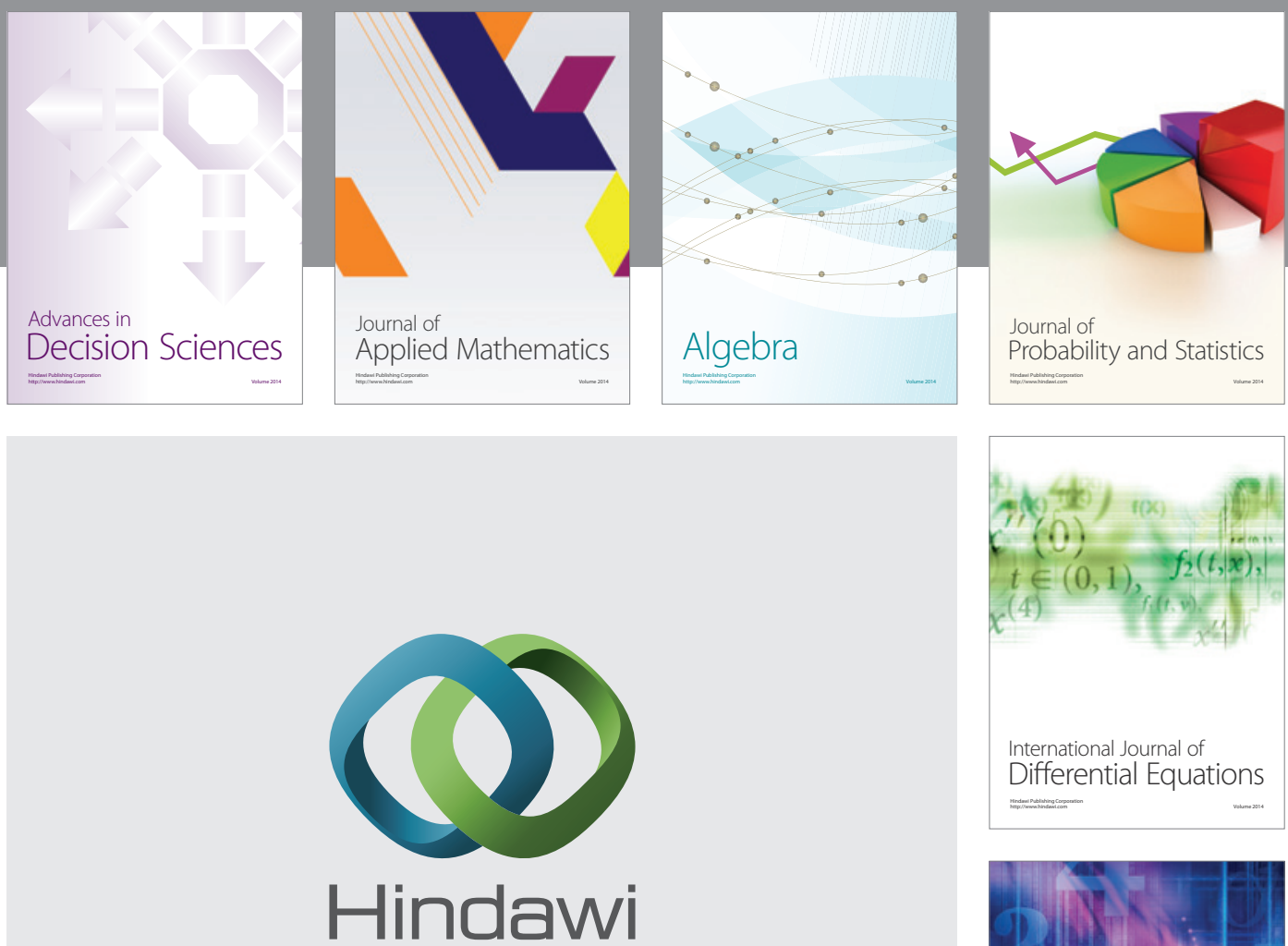

Submit your manuscripts at http://www.hindawi.com
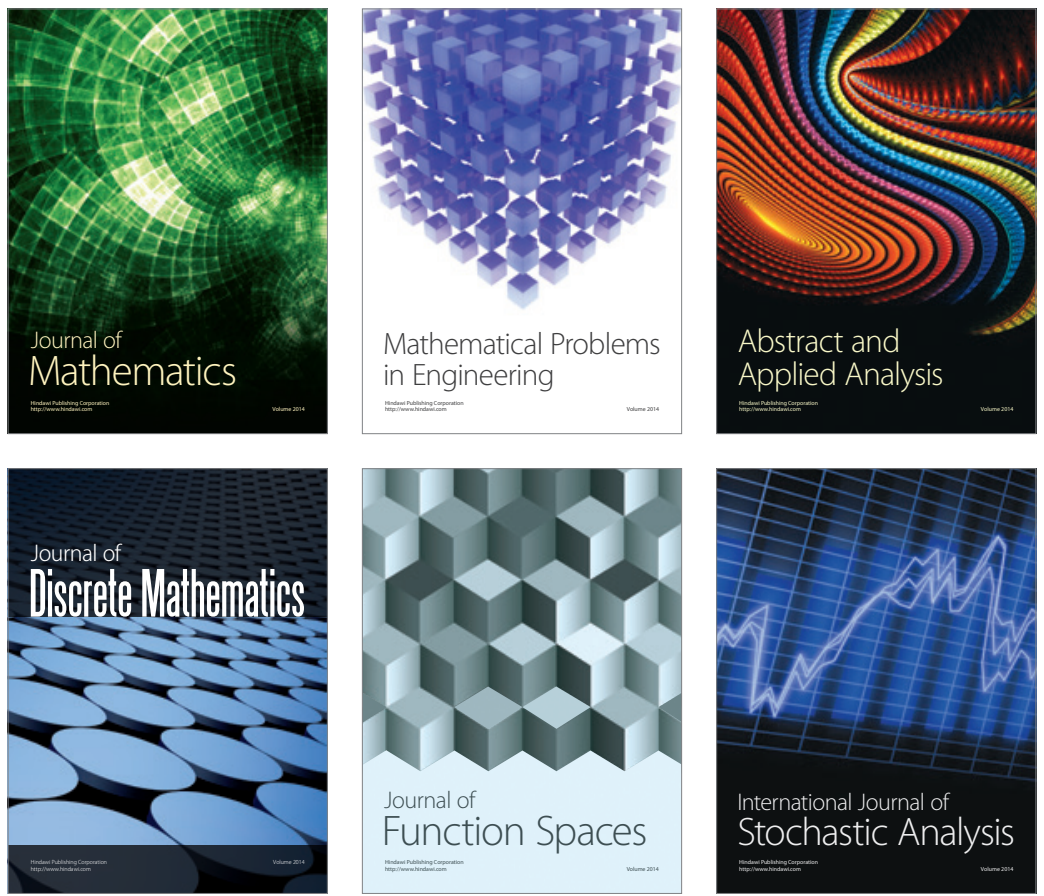

Journal of

Function Spaces

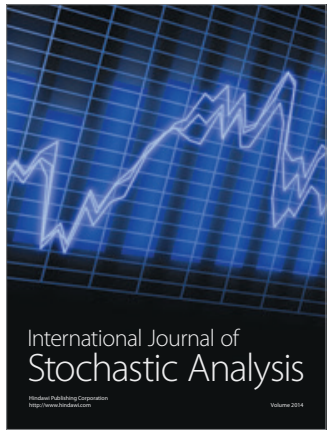

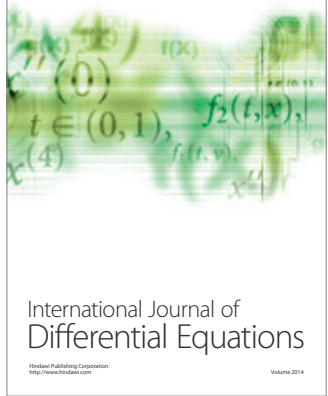
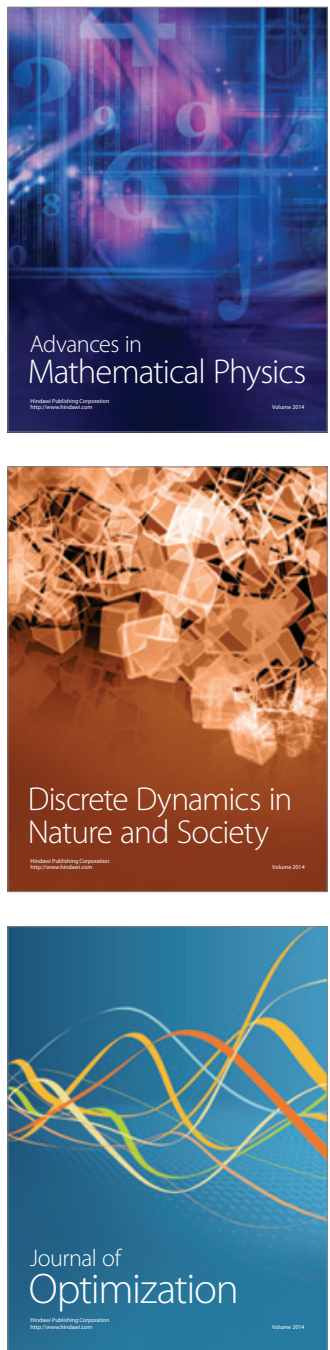